an important one in the development of A.C. transmission. Alternators, too, have become more complicated by the introduction of hydrogen cooling and special excitation devices to obtain maximum stability of operation with machines of minimum weight per unit of output.

$R$. Langlois-Berthelot gives a clear and interesting account of both classes of plant, which is designed to serve advanced students in technical schools and engineers who have to operate and maintain such plant. He puts his wide experience as a constructor and as a system engineer frankly at the service of his readers, and it would be difficult to find so much practical information in any other single volume. As it is impossible entirely to segregate the problems involved in operating alternators and transformers on an interconnected network, he concludes with an introduction to the study of such networks. This outlines the problems of steady-state operation, short-circuit conditions, stability, surge voltages, power-flow regulation, and optimum utilization of plant from the point of view of duration of life.

All who are concerned with large-scale electricity supply will find the author's treatment to be eminently practical and stimulating. His method, which uses more words than illustrations, will not appeal to many British engineers; but it is not without merit when the words are skilfully used, as is the case in this instance.

C. W. Marshall

\section{THEORY OF METALS FOR THE PRACTICAL METALLURGIST}

Electrons, Atoms, Metals and Alloys

By Dr. William Hume-Rothery. Pp. 377. (London : Cliffe and Sons, Ltd., 1948.) 25s. net.

7 HIS book has been written with a particular purpose--to introduce the latest advances in the theory of metals to the practical metallurgist. No one could be better suited to this task than Dr. W. Hume-Rothery, who has made so many important contributions to the subject, and who has yet kept his feet firmly planted on the ground of experimental work. In order to be able to lay stress on the more difficult points, and to anticipate difficulties that may arise in the mind of the practical metallurgist, the author has written the book in the form of a dialogue between a "Young Scientist" (a very able fellow) and an "Older Metallurgist" (a remarkably intelligent person who knows all the right questions to ask).

It is doubtful, however, whether the book will serve the purpose intended; the range of subjectmatter is so wide that the ordinary reader, having been conducted through the maze of the electronic structures of the elements (all of them), wave mechanies, free-electron theory, Brillouin zones, electron compounds and nuclear physics, may well be almost as bewildered at the end of the book as at the beginning. Pruning would probably improve the book considerably; general principles could have been emphasized at the expense of completeness, and some sections, such as those on plastic deformation and nuclear physics, could have been omitted completely.

However, it is quite possible that the book will be of considerable value to people other than those for whom it is intended. Those who still find a mental picture useful in absorbing now ideas-and if, in addition, they have to deal with students of the same frame of mind-may often be glad to have this book to turn to for reference. Theorists may protest that the representations are often too precise, but, nevertheless, they are better than the complete absence of representation that many people would have to be content with if they had to rely upon the mathematics alone.

After these general comments, it may seem odd to pick out a single sentence for comment. But why, on p. 64, has Dr. Hume-Rothery stated so emphatically, and apparently unnecessarily, that "It is not the province of Science to answer the question "Why ?" "? How much of modern science would have existed had not so many people been actuated by this word?

\section{MONOGRAPH ON THE BANANA}

\section{Bananas}

Chemistry, Physiology, Technology. By Harry W. von Loesecke. (Economic Crops, Vol. 1.) Pp. xi+ 189. (Now York and London: Interscience Publishers, Inc., 1949.) $36 s$.

THIS is the first of a series of volumes in which food crops will be given monographic treatment, special attention being paid to their chemical composition and biochemical aspects. This venture, which is justified by the accumulation of analytical data during recent decades, has much to commend it and will be appreciated by those who have to cope with the rapidly growing, but varied and often scattered, literature relating to the major crops. In general, the aim is to assemble and integrate research data relating to the many aspects of the production and the utilization of crops.

In this volume the author has cast his net widely, and within less than two hundred pages has dealt in some detail with the many interesting themes and problems presented by the banana-a major tropical crop and one requiring special care during the postharvesting phase of refrigerated transport and controlled ripening. Individual chapters are devoted to the history of the banana industry, the structure, development and culture of the plant, the commercial storage and ripening of the fruit, with special reference to the related physiological and biochemical problems, the commercial utilization of banana products, their nutritive value, the diseases and pests that beset the crop, and statistics of the industry in different parts of the world. To eover this ground the author has had to refer to a wide range of literature, some of a rather general nature, but much of it, for example, the physiology and biochemistry of ripening fruit, of a highly specialized kind. In the chapter on the physiology of ripening fruits, a large volume of research results has been brought together for the first time.

Although it is possible to point to some omissions in the text, this work undoubtedly provides a valuable, condensed survey of information on the banana and should accordingly serve the purpose for which it is intended, that is, to enable research workers to become cognizant of what has already been achieved in this varied and interesting field.

C. W. WARDIAW 